\title{
Position-dependent mass in strong quantum gravitational background fields.
}

\author{
Latévi Mohamed Lawson \\ ${ }^{1}$ African Institute for Mathematical Sciences (AIMS) Ghana \\ Summerhill Estates, East Legon Hills, Santoe, Accra \\ P.O. Box LG DTD 20046, Legon, Accra, Ghana \\ ${ }^{2}$ Université de Lomé, Faculté des Sciences, Département de Physique, \\ Laboratoire de Physique des Matériaux et des Composants \\ à Semi-Conducteurs, 01 BP 1515 Lomé, Togo \\ latevi@aims.edu.gh
}

February 1, 2022

\begin{abstract}
More recently, we have proposed a set of noncommutative space that describes the quantum gravity at the Planck scale [J. Phys. A: Math. Theor. 53, 115303 (2020)]. The interesting significant result we found is that, the generalized uncertainty principle induces a maximal measurable length of quantum gravity. This measurement revealed strong quantum gravitational effects at this scale and predicted a detection of gravity particles with low energies. In the present paper, to make evidence this prediction, we study in this space, the dynamics of a particle with position-dependent mass (PDM) trapped in an infinite square well. We show that, by increasing the quantum gravitational effect, the PDM of the particle increases and induces deformations of the quantum energy levels. These deformations are more pronounced as one increases the quantum levels allowing, the particle to jump from one state to another with low energies and with high probability densities.
\end{abstract}

Keywords: Generalized Uncertainty Principle; Minimal and maximal lengths of quantum gravity; Position-dependent mass; Quantum infinite square well

\section{Introduction}

Max Planck's length $l_{p}$ is conjectured to be the smallest length of nature where the quantum gravity effects are sensible. It is about $l_{p}=10^{-35} \mathrm{~m}$ beyond which space-time distances cannot be resolved. All quantum gravity theories, such as string theory [2] and 
loop quantum gravity [3] particularly accounts for the existence of a minimal measurable length $\Delta x$ which is proportional to the Planck length $l_{p}$. To theoretically realize this minimal length scale, a simple model has been introduced, the so-called Generalized Uncertainty Principle (GUP) [4, 5, 6, 7, 8, 9, 10, 11, 12, 13, 14, 15, 16, 17, 18] which is a gravitational correction to quantum mechanics. Moreover, to test this length in practice, many physicists believe that it is not possible, because it needs extremely high energies for its detection. For my point of view as some other physicists [19, 20, 21, 22, 23], the measurement of this length scale is somewhat difficult, but not impossible. To circumvent this problem of high energy requirement, we recently proposed in two dimensions (2D) a set of noncommutative algebra [1] that makes quantum gravitational measurement stronger at the Planck scale. This measurement is manifested by the existence of maximal length scale $l_{\max }$ which is consistent with the one proposed by Perivolaropoulos [23].

One of the biggest properties of gravity in general relativity (GR) is that, the gravitational field becomes stronger for heavy systems that curve the space, allowing the surronding light systems to fall down with low energies. As space-time is distorted in a gravitational field, Einstein has shown that relativistic effects such as time dilation and length contraction take effect. Such properties of gravity can also be manifested in quantum mechanics (QM). Thus, based on the latter properties, one can postulate that, strong quantum gravitational fields can increase the mass of particles in a certain volume in such a way that, the variation of their masses can be measured. To theoretically realize this process, we consider a quantum system whose mass changes with the position. Such a system is well known in the literature [23, 24, 25, 26, 27, 28, 29] and is used to describe the dynamics of particles in semiconductor heterostructures materials [30, 31, 32, 33]. In the present situation, we trap this system in an infinite square well potential in which the quantum gravitational background fields are strong. Then, we study the spectrum of this system by solving analytically the Schrödinger equation. Firstly, we observe that the states of the system exhibit properties similar to Gaussian states of the standard QM which are consequences of quantum fluctuations at this scale. Secondly, we remark that, the energy spectrum of this system is weakly proportional to the ordinary one of quantum mechanics free of the gravitational theory. This indicates that the effects of quantum gravity in this well increase the PDM which consequently induces deformations of energy levels so as to enable the particle to jump from one state into another with low energies and with high probability densities.

This paper is organised as follows: In the next section, we review in 1D, the outgoing of this GUP model with strong quantum gravity measurement and the corresponding local translation generator. Section 3 is devoted to the study of PDM in an infinite square-well potential in strong quantum gravitational background fields. We give the spectrum of this system by solving analytically the Schrödinger equation. Finally, we conclude this work in section 4 . 


\section{GUP with maximal length and its deformed translation symmetry}

In this section, we review the 1D GUP model with maximal length and its corresponding local translation generator.

\subsection{GUP with maximal length}

Let consider a set of symmetric operators $\hat{X}, \hat{Y}, \hat{P}_{x}, \hat{P}_{y}$ defined on the 2D Hilbert space $\mathcal{H}_{\theta \tau}=\mathcal{L}^{2}\left(\mathbb{R}^{2}\right)$ which satisfy the following commutation relations and all possible permutations of the Jacobi identities [1]

$$
\begin{aligned}
{[\hat{X}, \hat{Y}] } & =i \theta\left(\mathbb{I}-\tau \hat{Y}+\tau^{2} \hat{Y}^{2}\right), \quad\left[\hat{X}, \hat{P}_{x}\right]=i \hbar\left(\mathbb{I}-\tau \hat{Y}+\tau^{2} \hat{Y}^{2}\right), \\
{\left[\hat{Y}, \hat{P}_{y}\right] } & =i \hbar\left(\mathbb{I}-\tau \hat{Y}+\tau^{2} \hat{Y}^{2}\right), \quad\left[\hat{P}_{x}, \hat{P}_{y}\right]=0, \\
{\left[\hat{Y}, \hat{P}_{x}\right] } & =0, \quad\left[\hat{X}, \hat{P}_{y}\right]=i \hbar \tau(2 \tau \hat{Y} \hat{X}-\hat{X})+i \theta \tau\left(2 \tau \hat{Y} \hat{P}_{y}-\hat{P}_{y}\right),
\end{aligned}
$$

where $\theta, \tau \in \mathbb{R}_{+}^{*}$ are both deformed parameters that describe the Planck scale. The parameter $\tau$ is the GUP deformed parameter [6, 7, 8] related to quantum gravitational effects while the parameter $\theta$ is related to the noncommutativity of the space [9, 34] at this scale. In the framework of noncommutative classical or quantum mechanics, the parameter $\theta$ is proportional to the inverse of constant magnetic fields $B(\theta \sim 1 / B)$ [35, 36, 37. Since the algebra (1) describes the space at the Planck scale, then such magnefic fields are necessarily superstrong and may play the role of primordial magnetic fields. We notice that [1] both parameters are unified as a minimal length scale $\Delta X_{\min }=$ $l_{\min }=\tau \theta=\tau / B$. Futhermore, this minimal length measure $\Delta X_{\min }$ is coupled with the maximal length $\Delta Y_{\max }=l_{\max }=\tau^{-1}$ by the inverse of strong magnetic fields $B$ as follows

$$
\Delta X \Delta Y=\frac{1}{B}=l_{\min } l_{\max }
$$

At the singulary point $l_{\min }$ results of unification of magnetic fields and quantum gravitational fields. Since the Planck length scale marks the frontier of our universe, the origine of magnetic fields at this scale is conjectured to be from multiverse that bounces at this minimal position $l_{\text {min }}$. As predicted in our previous work [1], this scenario breaks up the big bang singularity. This part of the paper is currently under investigation and will be the object of further exhaustive study. However, simultaneous measurements of operators $\left(\hat{X}, \hat{P}_{x}\right),\left(\hat{X}, \hat{P}_{y}\right),\left(\hat{P}_{x}, \hat{P}_{y}\right)$ and $\left(\hat{Y}, \hat{P}_{x}\right)$ are spatial isotropy since their measurements do not present any minimal/maximal length or minimal momentum. Finally, for the case of interest in this paper i.e a simultaneous $\hat{Y}, \hat{P}_{y}$-measurement contains information on how the properties of gravity in QM are similar to the ones of GR.

The one dimensional set of the algebra (1) becomes

$$
[\hat{X}, \hat{P}]=i \hbar\left(\mathbb{I}-\tau \hat{X}+\tau^{2} \hat{X}^{2}\right),
$$

where the operators $\hat{X}$ and $\hat{P}$ are defined on $\mathcal{H}_{\tau}=\mathcal{L}^{2}(\mathbb{R})$. This proposal 3 follows the recent approach of quantum gravity measurement introduced by Perivolaropoulos 
[23. It has been shown that quantum gravity naturally arises from cosmology due to the presence of particle horizons with the parameter $\alpha=\alpha_{0} \frac{H_{0}^{2}}{c^{2}}$ where $H_{0}$ is the Hubble constant, $c$ is the speed of light and $\alpha_{0}$ is a dimensionless parameter.

From the commutation relation (3), an interesting feature can be observed through the following uncertainty relation:

$$
\Delta X \Delta P \geq \frac{\hbar}{2}\left(1-\tau\langle\hat{X}\rangle+\tau^{2}\left\langle\hat{X}^{2}\right\rangle\right)
$$

Using the relation $\left\langle\hat{X}^{2}\right\rangle=\Delta X^{2}+\langle\hat{X}\rangle^{2}$, equation (4) can be rewritten as a second order equation for $\Delta X$. The solutions are

$$
\Delta X=\frac{\Delta P}{\hbar \tau^{2}} \pm \sqrt{\left(\frac{\Delta P}{\hbar \tau^{2}}\right)^{2}-\frac{\langle\hat{X}\rangle}{\tau}(\tau\langle\hat{X}\rangle-1)-\frac{1}{\tau^{2}}} .
$$

This equation leads to the absolute minimal uncertainty $\Delta P_{\min }$ in $P$-direction and the absolute maximal uncertainty $\Delta X_{\max }$ in $X$-direction for $\langle\hat{X}\rangle=0$

$$
\Delta X_{\max }=l_{\max }=\frac{1}{\tau} \quad \text { and } \quad \Delta P_{\min }=\hbar \tau .
$$

It is well known [6] that, the existence of minimal uncertainty raised the question of singularity of the space i.e the space is inevitably bounded by minimal quantity beyond which any further localization of particle is not possible. In the presence situation, the minimal momentum $\Delta P_{\text {min }}$ leads to the lost of representation in $P$-direction whereas a maximal measurement $\Delta X_{\max }$ is the physical space of wavefunction representations i.e all functions $\psi(X) \in \mathcal{H}_{\tau}=\mathcal{L}^{2}(-\infty,+\infty)$ vanish at the boundary $\psi(-\infty)=0=\psi(+\infty)$.

From the uncertainty relation (4), it is interesting to observe that, the GUP is reduced into $\Delta X \Delta P \sim \hbar$. As is well known from the Heisenberg principle, the latter relation can be casted into the form

$$
\Delta X \Delta E \sim \hbar c \Longrightarrow \Delta E \sim \frac{\hbar c}{\Delta X},
$$

where $\Delta E \sim \Delta P c$. Unlike the results obtained in [4, 11, 12, 13, 14, here the required uncertainty energy is weak since the dimension of the length $\Delta X$ is very large. This indicates that a maximal localization of quantum gravity induces weak energies for its measurement. Let us now consider the equation $\Delta X=\Delta t c$. Inserting this equation in (7), one obtains

$$
\Delta t \sim \frac{\hbar}{\Delta E}
$$

Since, the uncertainty energy is low in this space due to the strong quantum gravitational effects, then its time $\Delta t$ strongly increases i.e the time runs more slowly in this space. In comparison with the minimal length theories, the concept of a strong quantum gravity developed in this paper admits a close analogy with the properties of gravity in GR 
whereas the scenario of minimal length describes an exotic space i.e a space with low quantum gravity for which its properties are out of the common sense.

One recovers the algebra (3) by setting the operators as follows

$$
\hat{X}=\hat{x}, \quad \hat{P}=\left(\mathbb{I}-\tau \hat{x}+\tau^{2} \hat{x}^{2}\right) \hat{p},
$$

where the symmetric operators $\hat{x}$ and $\hat{p}$ defined on $\mathcal{H}=\mathcal{L}^{2}(\mathbb{R})$ satisfy the Heisenberg algebra

$$
[\hat{x}, \hat{p}]=i \hbar .
$$

From the representation $(9)$, it follows that the position operator $\hat{X}$ is symmetric while the momentum operator $P$ is not. Thus, one observes

$$
\hat{X}^{\dagger}=\hat{X}, \quad \hat{P}^{\dagger}=\hat{P}+i \hbar \tau(\mathbb{I}-2 \tau \hat{X}) .
$$

In order to map this operator into symmetrical one, some synonymously concepts introduced in the literature can be used such as: the PT-symmetry [38, 39], the quasiHermiticity [40, 41], the pseudo-Hermiticity [42, 43, 44]. In the presente case, to guarantee the symmetry of the operator $\hat{P}$, we propose the following deformed completeness relation

$$
\int_{-\infty}^{+\infty} \frac{d x}{1-\tau x+\tau^{2} x^{2}}|x\rangle\langle x|=\mathbb{I} .
$$

By performing a partial integration, one can easily show that [1]

$$
\langle\phi \mid \hat{P} \psi\rangle=\left\langle\hat{P}^{\dagger} \phi \mid \hat{P} \psi\right\rangle \text {. }
$$

Consequently, the scalar product between two states $|\psi\rangle$ and $|\phi\rangle$ and the orthogonality of position eigenstate become

$$
\begin{aligned}
\langle\psi \mid \phi\rangle & =\int_{-\infty}^{+\infty} \frac{d x}{1-\tau x+\tau^{2} x^{2}} \psi^{*}(x) \phi(x), \\
\left\langle x \mid x^{\prime}\right\rangle & =\left(1-\tau x+\tau^{2} x^{2}\right) \delta\left(x-x^{\prime}\right) .
\end{aligned}
$$

\subsection{Deformed translation symmetry}

Einstein's theory of gravity is based on the symmetry principle of invariance under general coordinate transformations, seen as local space-time transformations generated by the local translation generators. In this background, the gravitational force can be modelled and described from a geometric point of view in terms of the curvature of space-time [22]. Therefore, in this framework, considering the configuration space wave function defined by $\psi(x):=\langle x \mid \psi\rangle$, the moment operator $\hat{P}$ acts on $\psi(x)$ as differential operator such as

$$
\begin{aligned}
\hat{P} \psi(x) & =-i \hbar D_{x} \psi(x), \\
\frac{i}{\hbar} \hat{P} \psi(x) & =D_{x} \psi(x)
\end{aligned}
$$


where $D_{x}=\left(1-\tau x+\tau^{2} x^{2}\right) \partial_{x}$ is the deformed partial derivative which introduces asymmetrical effects in the system. For the operator $\hat{P}$, one can associate through the exponential map a $\tau$-translation operator $\hat{\mathcal{T}}_{\tau}$ that induces an infinitesimal distance $\delta a$ in this direction

$$
\hat{\mathcal{T}}_{\tau}(\delta a)=\exp \left(\frac{i}{\hbar} \delta a \hat{P}\right), \quad \text { with } \quad \delta a \geq 0
$$

Since $\delta a$ is infinitesimal, we expand the operator $\hat{\mathcal{T}}_{\tau}$ to first order of $\delta a$ as follows

$$
\hat{\mathcal{T}}_{\tau}(\delta a)=\mathbb{I}+\frac{i}{\hbar} \delta a \hat{P} \quad \text { and } \quad \lim _{\delta a \rightarrow 0} \hat{\mathcal{T}}_{\tau}(\delta a)=\mathbb{I} .
$$

Since the operators $\hat{P}$ is symmetric, one can readly show that the $\tau$-translation operator $\hat{\mathcal{T}}_{\tau}$ forms a unitary group

$$
\hat{\mathcal{T}}_{\tau}^{\dagger}(\delta a) \hat{\mathcal{T}}_{\tau}(\delta a)=\mathbb{I}=\hat{\mathcal{T}}_{\tau}(\delta a) \hat{\mathcal{T}}_{\tau}^{\dagger}(\delta a) \quad \text { and } \quad \hat{\mathcal{T}}_{\tau}(0)=\mathbb{I},
$$

which implies

$$
\hat{\mathcal{T}}_{\tau}^{\dagger}(\delta a)=\hat{\mathcal{T}}_{\tau}^{-1}(\delta a)=\hat{\mathcal{T}}_{\tau}(-\delta a)=\mathbb{I}-\frac{i}{\hbar} \delta a \hat{P} .
$$

Consequently, for two arbitrary states $\left|\psi^{\prime}\right\rangle$ and $\left|\phi^{\prime}\right\rangle$ obtained from the action of $\hat{\mathcal{T}}_{\tau}$ on the respective states $|\psi\rangle$ and $|\phi\rangle$, such that

$$
\left|\psi^{\prime}\right\rangle=\hat{\mathcal{T}}_{\tau}(\delta a)|\psi\rangle, \quad\left|\phi^{\prime}\right\rangle=\hat{\mathcal{T}}_{\tau}(\delta a)|\phi\rangle,
$$

one can easily check that the inner product of both states is $\tau$-translational invariant

$$
\left\langle\phi^{\prime} \mid \psi^{\prime}\right\rangle=\langle\phi \mid \psi\rangle .
$$

The action of $\hat{\mathcal{T}}_{\tau}$ on the state $|x\rangle$, translates this state by the amount of $\delta a$ to the right

$$
\hat{\mathcal{T}}_{\tau}(\delta a)|x\rangle=\left|x-\delta a\left(1-\tau x+\tau^{2} x^{2}\right)\right\rangle .
$$

From this, it also follows that

$$
\langle x| \hat{\mathcal{T}}_{\tau}(\delta a)=\left\langle x+\delta a\left(1-\tau x+\tau^{2} x^{2}\right)\right| .
$$

An alternative way of seeing this result without relying on position eigenstates representation is by considering the following action

$$
\begin{aligned}
\left\langle x\left|\hat{\mathcal{T}}_{\tau}(\delta a)\right| \psi\right\rangle & =\psi(x)+\delta a D_{x} \psi(x), \\
& =\psi\left(x+\delta a\left(1-\tau x+\tau^{2} x^{2}\right)\right) .
\end{aligned}
$$

If the states of particles are moved by the amount $\delta a_{1}$ and $\delta a_{2}$, overall they have been moved by the amount $\delta a_{1}+\delta a_{2}$

$$
\hat{\mathcal{T}}_{\tau}\left(\delta a_{1}\right) \hat{\mathcal{T}}_{\tau}\left(\delta a_{2}\right) \psi(x)=\psi\left(x+\delta a_{1}\left(1-\tau x+\tau^{2} x^{2}\right)+\delta a_{2}\left(1-\tau x+\tau^{2} x^{2}\right)\right)
$$




$$
=\hat{\mathcal{T}}_{\tau}\left(\delta a_{1}+\delta a_{2}\right) \psi(x) .
$$

Let us consider $\hat{H}$, the operator Hamiltonian of a system defined within this space by

$$
\hat{H}(\hat{P}, \hat{X})=\frac{\hat{P}^{2}}{2 m_{0}}+V(\hat{X}),
$$

where $\mathrm{V}$ is the potential energy of the system. For periodical deformed potential energy

$$
\hat{\mathcal{T}}_{\tau}^{\dagger}(\delta a) V(\hat{x}) \hat{\mathcal{T}}_{\tau}(\delta a)=V\left(\hat{x}+\delta a\left(1-\tau \hat{x}+\tau^{2} \hat{x}^{2}\right)\right)=V(\hat{x}),
$$

the entire Hamiltonian satisfies

$$
\left[\hat{H}, \hat{\mathcal{T}}_{\tau}\right]=0 .
$$

The time-dependent deformed Schrödinger equation is

$$
-\frac{\hbar^{2}}{2 m_{0}} D_{x}^{2} \psi(x, t)+V(x, t) \psi(x, t)=i \hbar \partial_{t} \psi(x, t) .
$$

In analogy with problems involving PDM systems [24, 25, 26, 27, 28, 29, 30, this equation is rewritten as follows

$$
i \hbar \partial_{t} \psi(x, t)=\left[-\frac{\hbar^{2}}{2 m_{0}}\left(\sqrt{\frac{m_{0}}{m(x)}} \partial_{x}\right)^{2}+V(x)\right] \psi(x, t)
$$

where

$$
m(x)=\frac{m_{0}}{\left(1-\tau x+\tau^{2} x^{2}\right)^{2}},
$$

being the PDM's particle strongly pertubated by quantum gravity. Figure 1 illustrates the PDM as a function of the position $x(0<x<0.5)$. One observes that the effective mass $m(x)$ in this description increases with $\tau$. This indicates that quantum gravitational fields increase with the effective $m(x)$ of the system. In other words, by increasing the mass of the system or by just taking larger masses and bringing them into quantum states, one can make the quantum gravitational effects stronger for a measurement. This description was experimentally carried out to measure the gravitational field of significantly smaller source masses with remarkable technical skills of realisation [46]. Moreover, the increase of $m(x)$ with the values of $\tau$ observed in this system is in perfect analogy with the theory of GR where massive objects induce strong gravitational fields. As it will be shown in the next section, the increase of the PDM of a particle in an infinite square well potential curves the quantum levels, which consequently implies the decrease of energy amplitudes and lead to higher probability densities of particles at this scale.

The probability density $\rho(x, t)=|\psi(x, t)|^{2}$ obeys the continuity equation

$$
\frac{\partial \rho(x, t)}{\partial t}+D_{x} J_{\tau}(x, t)=0,
$$

where the current density is given by

$$
J_{\tau}(x, t)=\frac{\hbar\left(1-\tau x+\tau^{2} x^{2}\right)}{2 i m_{0}}\left(\psi^{*} \frac{d \psi}{d x}-\psi \frac{d \psi^{*}}{d x}\right) .
$$




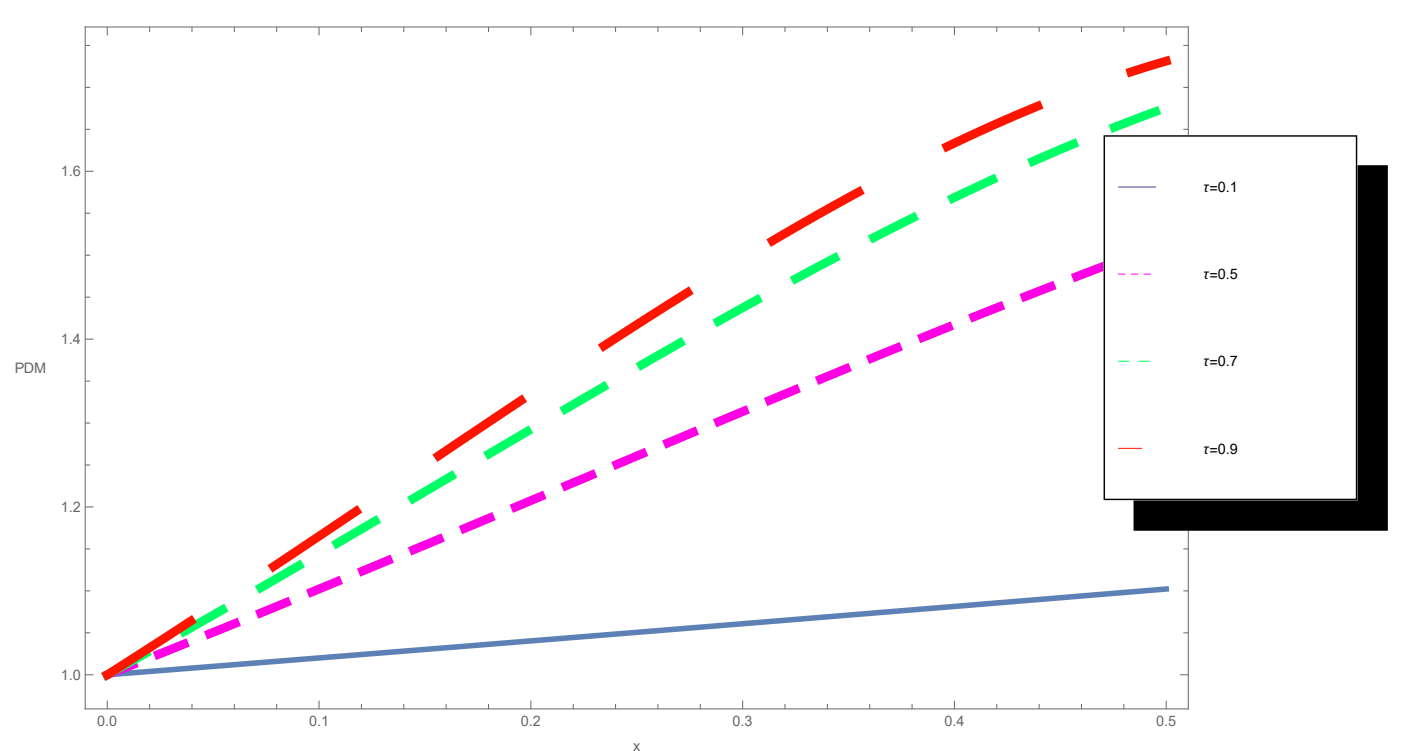

Figure 1: PDM $m(x)$ versus the position $x$ for different values of $\tau$

\section{$3 \quad$ PDM particle in an infinite square well}

\subsection{Eigensystems of PDM particle}

Let us consider the Hamiltonian of the above quantum system (28) confined in an infinite square-well potential, defined as

$$
V(x)=\left\{\begin{array}{l}
0, \quad 0<x<L, \\
\infty, \quad \text { otherwise }
\end{array}\right.
$$

For standing waves in a null potential, the wave function $\psi(x)$ satisfying equation (31) obeys

$$
-\frac{\hbar^{2}}{2 m_{0}} D_{x}^{2} \psi(x)=E \psi(x), \quad \text { with } \quad E>0
$$

or

$$
-\frac{\hbar^{2}}{2 m_{0}}\left[\left(1-\tau x+\tau^{2} x^{2}\right)^{2} \frac{d^{2}}{d x^{2}}-\tau(1-2 \tau x)\left(1-\tau x+\tau^{2} x^{2}\right) \frac{d}{d x}\right] \psi(x)=E \psi(x) .
$$

The solution of the equation (37) is given by

$$
\psi_{k}(x)=A \exp \left(i \frac{2 k}{\tau \sqrt{3}}\left[\arctan \left(\frac{2 \tau x-1}{\sqrt{3}}\right)+\frac{\pi}{6}\right]\right),
$$


where $k=\frac{\sqrt{2 m E}}{\hbar}$. Then by normalization $\left\langle\psi_{k} \mid \psi_{k}\right\rangle=1$, we have

$$
\begin{aligned}
1 & =\int_{0}^{L} \frac{d x}{1-\tau x+\tau^{2} x^{2}} \psi^{*}(x) \psi(x) \\
& =A^{2} \int_{0}^{L} \frac{d x}{1-\tau x+\tau^{2} x^{2}} .
\end{aligned}
$$

so, we find

$$
A=\sqrt{\frac{\tau \sqrt{3}}{2}}\left[\arctan \left(\frac{2 \tau L-1}{\sqrt{3}}\right)+\frac{\pi}{6}\right]^{-1 / 2} .
$$

Based on the reference [7, 47, the scalar product of the formal eigenstates is given by

$$
\left\langle\psi_{k^{\prime}} \mid \psi_{k}\right\rangle=\frac{\tau \sqrt{3}}{2\left(k-k^{\prime}\right)\left[\arctan \left(\frac{2 \tau L-1}{\sqrt{3}}\right)\right]} \sin \left(\frac{2\left(k-k^{\prime}\right)\left[\arctan \left(\frac{2 \tau L-1}{\sqrt{3}}\right)\right]}{\tau \sqrt{3}}\right) .
$$

This relation shows that, the normalized eigenstates $(39)$ are no longer orthogonal. However, if one tends $\left(k-k^{\prime}\right) \rightarrow \infty$, these states become orthogonal

$$
\lim _{\left(k-k^{\prime}\right) \rightarrow \infty}\left\langle\psi_{k^{\prime}} \mid \psi_{k}\right\rangle=0 .
$$

These properties show that, the states $\left|\psi_{k}\right\rangle$ are essentially Gaussians centered at $(k-$ $\left.k^{\prime}\right) \rightarrow 0$ (see Figure 2). This observation indicates quantum gravitational fluctuations at this scale.

We suppose that the wave function satisfies the Dirichlet condition i.e it vanishes at the boundaries $\psi(0)=0=\psi(L)$. Thus, using especially the boundary condition $\psi(0)=0$, the above wavefunctions $(39)$ becomes

$$
\psi_{k}(x)=A \sin \left(\frac{2 k}{\tau \sqrt{3}}\left[\arctan \left(\frac{2 \tau x-1}{\sqrt{3}}\right)+\frac{\pi}{6}\right]\right) .
$$

The quantization follows from the boundary condition $\psi(L)=0$ and leads to the equation

$$
\begin{aligned}
\frac{2 k_{n}}{\tau \sqrt{3}}\left[\arctan \left(\frac{2 \tau L-1}{\sqrt{3}}\right)+\frac{\pi}{6}\right] & =n \pi \quad \text { with } n \in \mathbb{N}^{*}, \\
k_{n} & =\frac{\pi \tau \sqrt{3} n}{2\left[\arctan \left(\frac{2 \tau L-1}{\sqrt{3}}\right)+\frac{\pi}{6}\right]} .
\end{aligned}
$$

Then, the energy spectrum of the particle is written as

$$
E_{n}=\frac{3 \pi^{2} \tau^{2} \hbar^{2} n^{2}}{8 m_{0}\left[\arctan \left(\frac{2 \tau L-1}{\sqrt{3}}\right)+\frac{\pi}{6}\right]^{2}}
$$




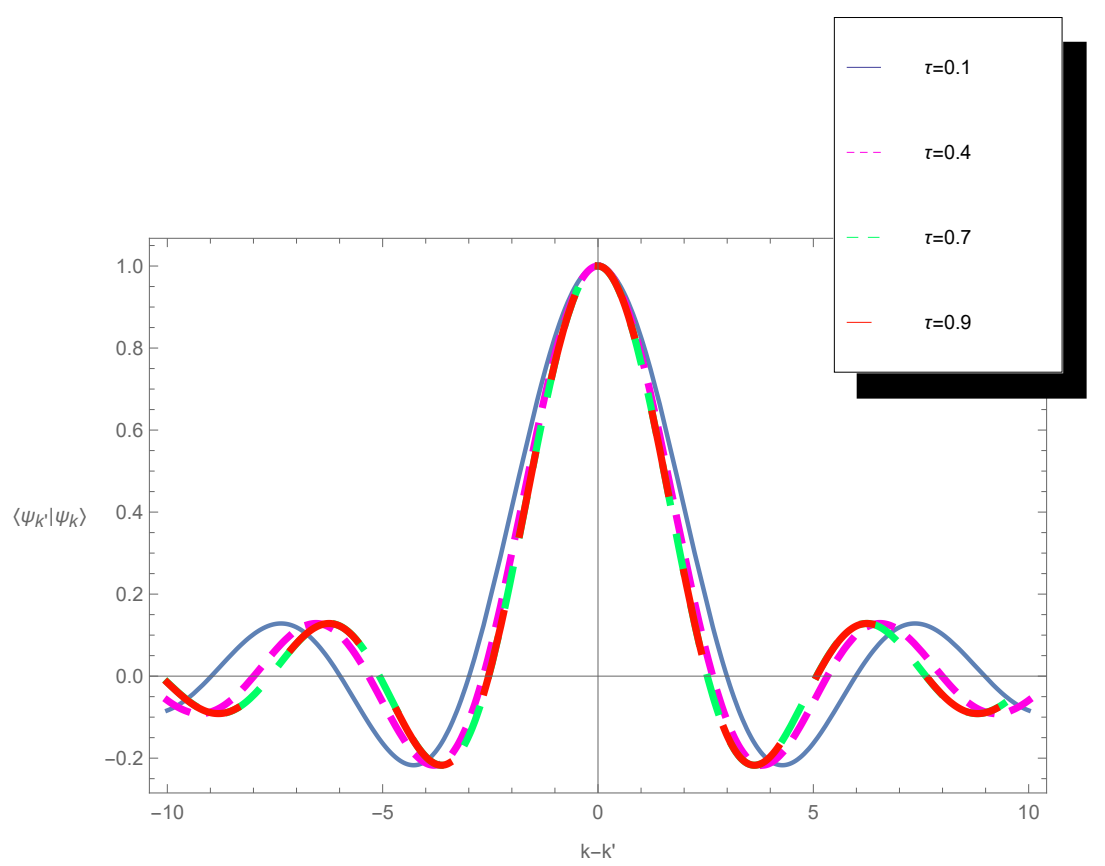

Figure 2: Variation of $\left\langle\psi_{k^{\prime}} \mid \psi_{k}\right\rangle$ versus $k-k^{\prime}$ with $L=1$

At the limit $\tau \rightarrow 0$, we have

$$
\lim _{\tau \rightarrow 0} E_{n}=\varepsilon_{n}=\frac{\pi^{2} \hbar^{2} n^{2}}{2 m_{0} L^{2}}
$$

where $\varepsilon_{n}$ is the spectrum of a free particle in an infinite square well potential of the basic quantum mechanics with the fundamental energy $\varepsilon_{1}=\frac{\hbar^{2} \pi^{2}}{2 m_{0} L^{2}}$. Thus, the energy levels can be rewritten as

$$
E_{n}=\frac{3}{4}\left[\frac{\tau L}{\arctan \left(\frac{2 \tau L-1}{\sqrt{3}}\right)+\frac{\pi}{6}}\right]^{2} \varepsilon_{n}<\varepsilon_{n} .
$$

Comparing this result (49) to various tandem of PDM systems [25, 27, 30], we realise that the parameters of deformations used in both approches have different implications. Here the effects of the parameter $\tau$ induces contractions of quantum levels which consequently lead to a decrease in the amplitude of the energy levels while the parameter of deformation in [25, 27, 30] leads to an increase in the energy levels of the particle.

The generalized wave function and the probability density corresponding to the en- 
ergies (47) are given by

$$
\begin{aligned}
& \psi_{n}(x)=A \sin \left(\frac{n \pi}{\left[\arctan \left(\frac{2 \tau L-1}{\sqrt{3}}\right)+\frac{\pi}{6}\right]}\left[\arctan \left(\frac{2 \tau x-1}{\sqrt{3}}\right)+\frac{\pi}{6}\right]\right), \\
& \rho_{n}(x)=A^{2} \sin ^{2}\left(\frac{n \pi}{\left[\arctan \left(\frac{2 \tau L-1}{\sqrt{3}}\right)+\frac{\pi}{6}\right]}\left[\arctan \left(\frac{2 \tau x-1}{\sqrt{3}}\right)+\frac{\pi}{6}\right]\right) .
\end{aligned}
$$

At the limit $\tau \rightarrow 0$, we have

$$
\begin{aligned}
\lim _{\tau \rightarrow 0} \psi_{n} & =\sqrt{\frac{2}{L}} \sin \left(\frac{n \pi}{L} x\right), \\
\lim _{\tau \rightarrow 0} \rho_{n} & =\frac{2}{L} \sin ^{2}\left(\frac{n \pi}{L} x\right) .
\end{aligned}
$$

\subsection{Numerical results and discussions}

In this part, numerical results and discussions have been done to investigate the effect of the parameter $\tau$ on the amplitudes of energy levels, on the wave functions and on the probability densities. For the calculations, we set $m=1, \hbar=1$ and $L=1$.

Figure 3 illustrates the energy levels of the particle as functions of the quantum number $n$ and the quantum gravity parameter $\tau$. Figure $3 a$ shows a plot of energy levels versus the quantum number $n$ for different values of $\tau$. Conversly to the graph obtained in [25, 27, 30], one observes that, the amplitudes of energy levels $E_{n} / \varepsilon_{1}$ decrease when $\tau$ increases. This is confirmed by the analytical result obtained in (49). The same reasoning can be applied to the energy levels versus the parameter $\tau$ for different values of $n$ (Figure $3 p$ ). One observes how the increase of $\tau$ gradually deforms the energy levels as one increases the quantum number $n$ from the fundamental level. These deformations curve the quantum levels, allowing particle to jump from one state to another with low energies. Moreover, the deformations observed at these quantum levels are in perfect analogy with the theory of GR where the gravity induces deformation of the space.

Figure 4 illustrates the eigenfunctions $\psi_{n}(x)$ and their respective probability densities $\rho_{n}(x)$ for the three lowest states $n=1, n=2, n=3$ for some values of the parameter $\tau \in\{0 ; 0.1 ; 0.2 ; 0.3\}$. As expected, increase of $n$ confines, shrinks and contracts $\psi_{n}(x)$ and $\rho_{n}(x)$. This fact comes to confirm the fundamental property of gravity which consists of length contraction.

The theory developed here can be easily extended to two and three dimensions. For example, when considering a square section of an infinite well, the corresponding wave function can be expressed as the product $\Psi_{n}(x, y)=\psi_{n}(x) \psi_{n}(y)$, where $\psi_{n}(x)$ and $\psi_{n}(y)$ are the wave functions in the $x$ and $y$ directions, respectively. The probability density $\rho_{n}(x, y)$ of a particle moving in a two-dimensional box is shown in Figure 5 such as in (a): $n_{x}=n_{y}=1$, (b) $n_{x}=n_{y}=2$, (c) for $n_{x}=n_{y}=3$ and in (d) $n_{x}=n_{y}=10$ where we have used $\tau=0.1$ in all panels. Evidently, as pointed out through the figure (4), it can be seen that for the first three lower states the probability to find a particle is practically 


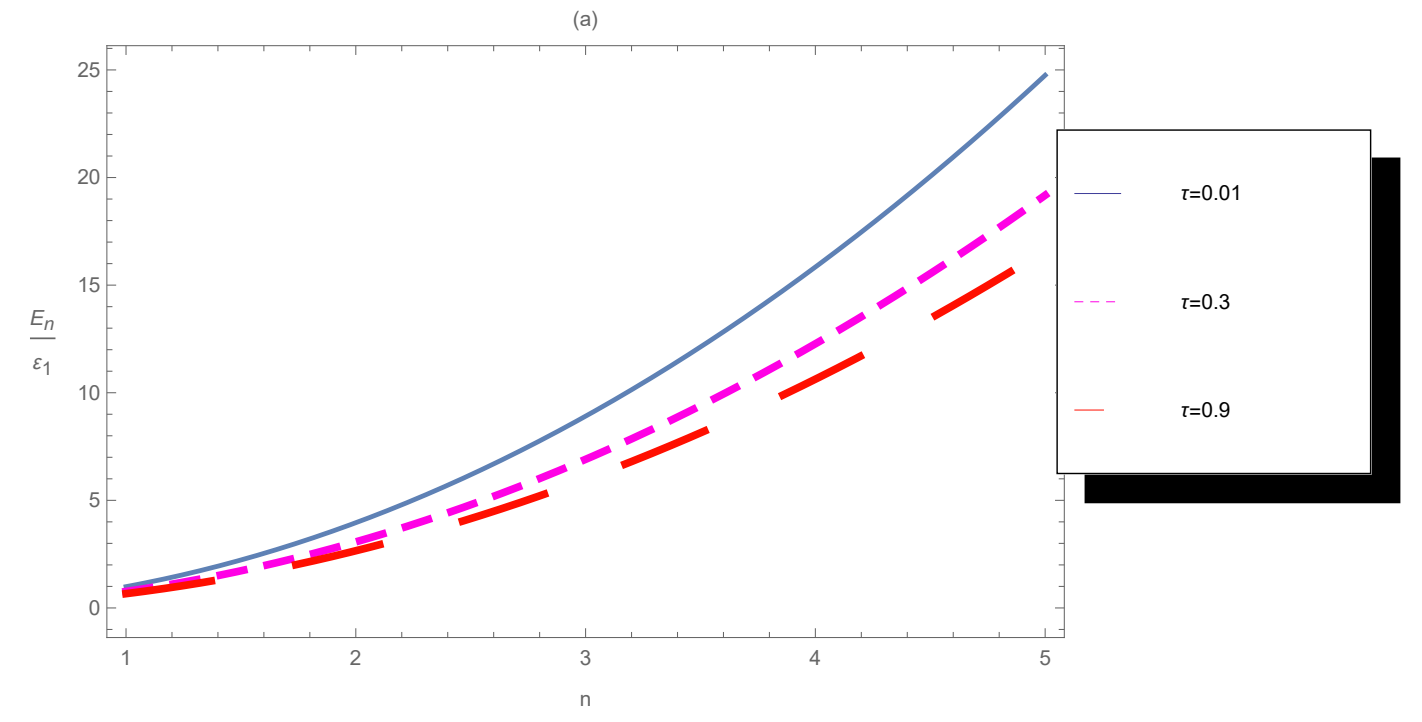

(b)

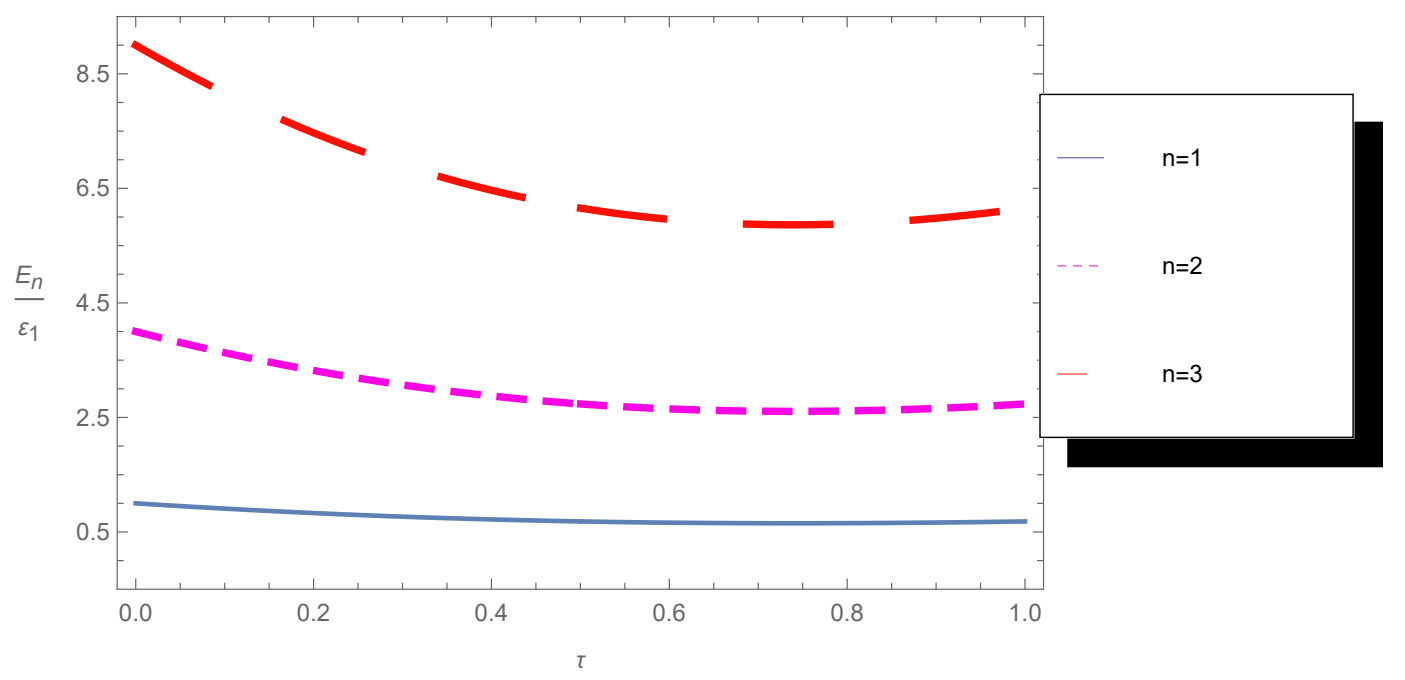

Figure 3: The energy $E_{n} / \varepsilon_{1}$ of the particle in $1 D$ box of length $L=1$ with mass $m=1$ and $\hbar=1$. 

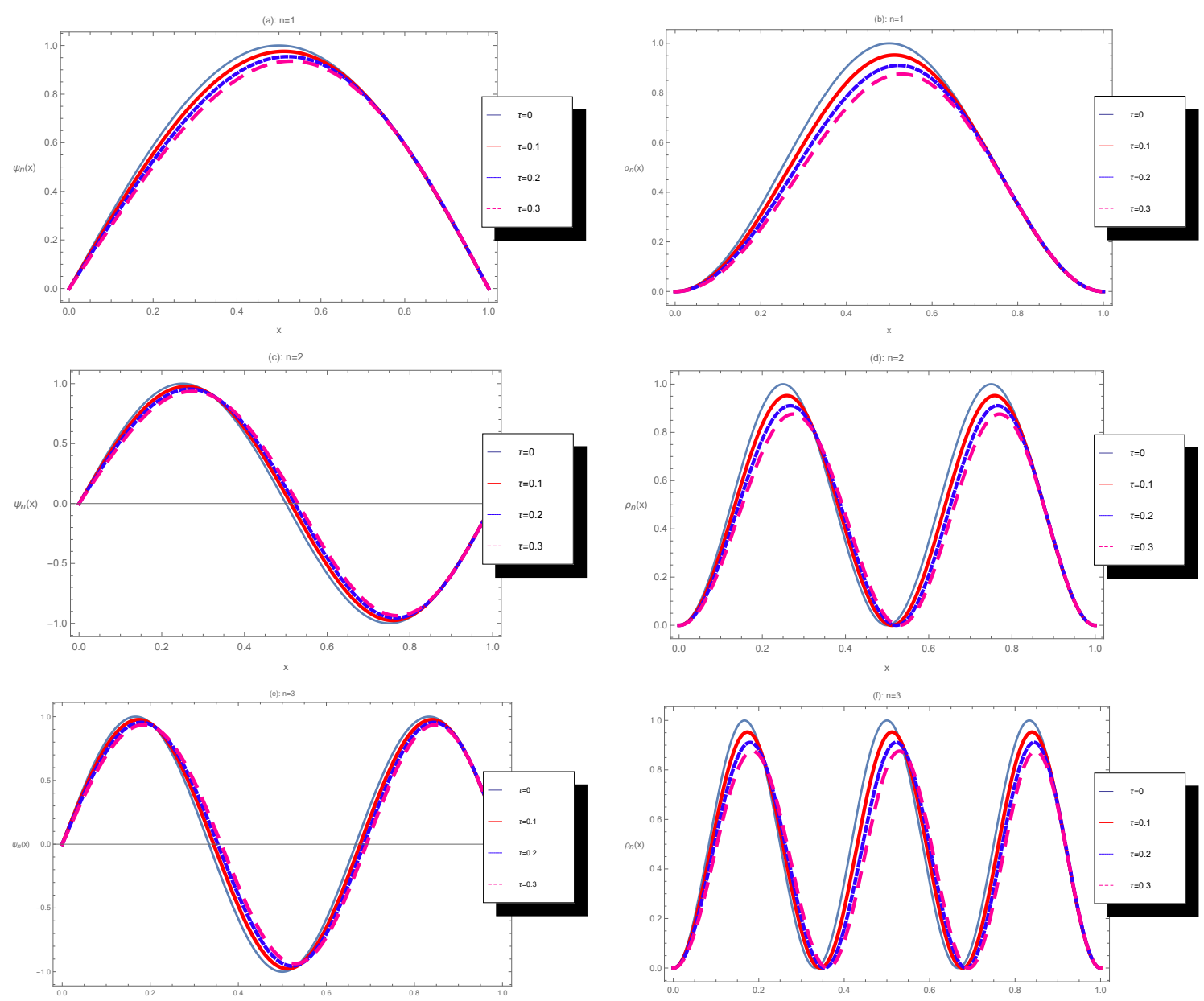

Figure 4: Wave functions $\psi_{n}(x)$ (left column) and probability densities $\rho_{n}(x)$ (right column) for a particle confined in an infinite square well of length $L=1$ deformed by the gravity parameter $\tau$. [(a) and (b)] $n=1$ (ground state), [(c) and (d)] $n=2$ (first excited state), [(e) and (f)] $n=3$ (second excited state) 
the same everywhere in the square well and this probability strongly increases with the quantum numbers. But, unlike the figure reported in [25, 27], here for large quantum numbers $n_{x}=n_{y}=10$, one observes a decrease of probability densities.
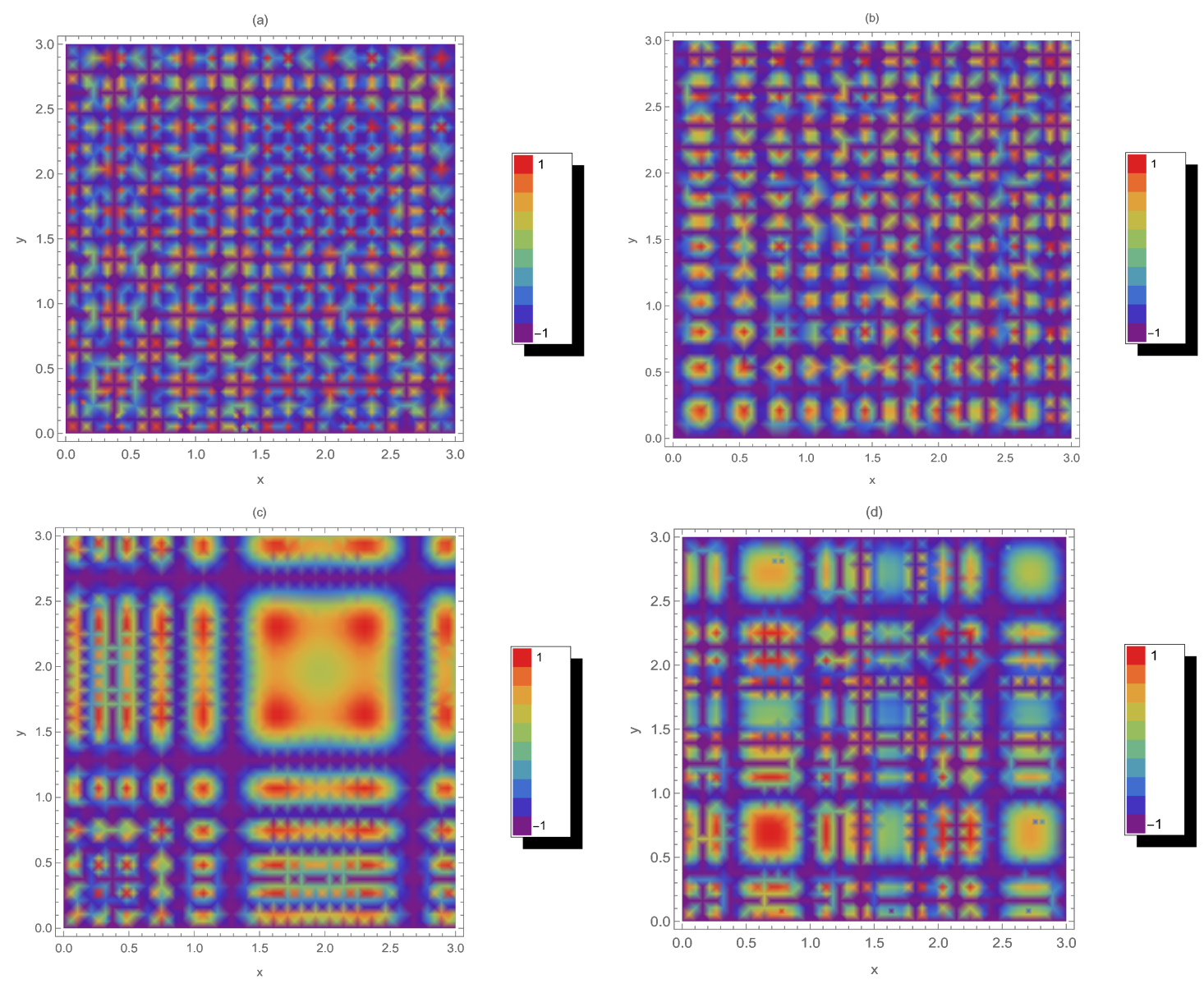

Figure 5: The probability density of a two-dimensional infinite square well for (a) $n_{x}=n_{y}=1$, (b) $n_{x}=n_{y}=2$, (c) $n_{x}=n_{y}=3$, and (d) $n_{x}=n_{y}=10$.

\section{Conclusion remarks}

In this paper, we study the dynamic of a position-dependent mass (PDM) trapped in an infinite square well potential. The increase of quantum gravitational background fields of this well has increased the PDM which in returned has induced deformations of quantum energy levels. These deformations are more pronounced as one increases the quantum levels allowing the particle to jump from one state to another with low energies and with high probability densities. Furthermore, we have observed that the states of this system exhibit properties similar to Gaussian states of the standard quantum mechanics 
which are consequences of quantum gravitational fluctuations at this scale. As expected, the increase of quantum numbers $n$ has confined, shrinked and contracted the states of this system. This characterization of quantum gravitational effects in this well is in perfect analogy with the one of general relativity (GR).

Moreover, it has been found that the mathematical foundations of the PDM systems are related to some generalized algebraic structures such as the $q$-deformed algebra [25, 48, 49] and the $\kappa$-deformed algebra [50, 51, 52]. Thus, this version of a PDM system introduced in this paper could give birth to a new algebraic structure namely the $\tau$-deformed algebra. This situation and the problem of harmonic oscillator could be investigated in this framework.

\section{Acknowledgments}

The author acknowledges support from AIMS-Ghana under the Postdoctoral fellow/teaching assistance (Tutor) grant

\section{References}

[1] L. Lawson, Minimal and maximal lengths from position-dependent noncommutativity, J. Phys. A: Math. Theor. 53, 115303 (2020)

[2] D. Amati, M. Ciafaloni and G. Veneziano, Can Space-Time Be Probed Below the String Size?, Phys.Lett. B 216, 41-47 (1989)

[3] C. Rovelli and L. Smolin, Discreteness of area and volume in quantum gravity, Nucl. Phys. B. 442, 593-619 (1995).

[4] M. Maggiore, A generalized uncertainty principle in quantum gravity, Phys. Lett. B 304, (1993) 65.

[5] M. Maggiore, Quantum groups, gravity, and the generalized uncertainty principle, Phys. Rev. D 49, 5182 (1994)

[6] A. Kempf, G. Mangano and R. Mann, Hilbert space representation of the minimal length uncertainty relation, Phys. Rev. D 52, 1108 (1995)

[7] A. Kempf and G. Mangano, Minimal length uncertainty relation and ultraviolet regularization, Phys. Rev. D. 55, 7909-7920 (1997)

[8] A. Kempf, Uncertainty relation in quantum mechanics with quantum group symmetry, J. Math. Phys. 35, (1994) 4483-4496.

[9] A. Fring, L. Gouba and F. Scholtz, Strings from position-dependent noncommutativity, J. Phys. A: Math. Theor. 43, 345401 (2010) 
[10] L. Lawson, L. Gouba and G. Avossevou, Two-dimensional noncommutative gravitational quantum well, J. Phys. A: Math. Theor. 50, 475202 (2017)

[11] F. Scardiglia and R. Casadio, Gravitational tests of the Generalized Uncertainty Principle, Eur. Phys. J. C 75, 425 (2015).

[12] S. Das and E. Vagenas, Universality of Quantum Gravity Corrections, Phys. Rev. Lett. 101, 221301

[13] G. Lambiase and F. Scardigli, Lorentz violation and generalized uncertainty principle, Phys. Rev. D 97, 075003 (2018)

[14] F. Brau and F. Buisseret, Minimal length uncertainty relation and gravitational quantum well, Phys. Rev. D 74, 0360002 (2006)

[15] G. Luciano, L. Petruzziello, Generalized uncertainty principle and its implications on geometric phases in quantum mechanics, Eur. Phys. J. Plus 136, 179 (2021)

[16] G. Luciano and L. Petruzziello, GUP parameter from maximal acceleration, Eur. Phys. J. C 79, 283 (2019)

[17] L. Buoninfante, G. Lambiase, G. Luciano and L. Petruzziello, Phenomenology of GUP stars, Eur. Phys. J. C 80, 853 (2020)

[18] L. Buoninfante, G. Luciano and L. Petruzziello, Generalized uncertainty principle and corpuscular gravity, Eur. Phys. J. C 79, 663 (2019)

[19] J. Schmöle, M. Dragosits, H. Hepach and M. Aspelmeyer, A micromechanical proof of principle experiment for measuring the gravitational force of milligram masses, Class. Quantum Grav. 33, 125031 (2016)

[20] I. Pikovski, M. R. Vanner, M. Aspelmeyer, M. Kim, and C. Brukner Probing Planckscale physics with quantum optics, Nat. Phys. 8, 393 (2012)

[21] M. Bawaj, C. Biancofiore et al.Probing deformed commutators with macroscopic harmonic oscillators, Nat Commun 6, 7503 (2015)

[22] D. Gao and M. Zhan, Constraining the generalized uncertainty principle with cold atoms, Phys. Rev. A 94, 013607 (2016).

[23] L. Perivolaropoulos, Cosmological horizons, uncertainty principle, and maximum length quantum mechanics, Phys. Rev. D 95, 103523 (2017)

[24] Oldwig von Roos, Position-dependent effective masses in semiconductor theory, Phys Rev B 27, 12 (1983)

[25] R. N. Costa Filho, M. Almeida, G. Farias, and J. Andrade Jr., Displacement operator for quantum systems with position-dependent mass, Phys Rev A, 84, 050102 (2011) 
[26] B. da Costa and E. Borges, Generalized space and linear momentum operators in quantum mechanics, J. Math Phys 55, 062105 (2014)

[27] S. Habib Mazharimousavi, Revisiting the displacement operator for quantum systems with position-dependent mass, Phys Rev A, 85, 034102 (2012)

[28] G. Bruno da Costa and Ernesto P. Borges, A position-dependent mass harmonic oscillator and deformed space, J. Math. Phys. 59, 042101 (2018)

[29] M. Rego-Monteiro and F. Nobre, Classical field theory for a non-Hermitian Schrodinger equation with position-dependent masses, Phys Rev A 88, 032105 (2013)

[30] B. da Costa, I. Gomez, and M. Portesi, $\kappa$-Deformed quantum and classical mechanics for a system with position-dependent effective mass, J. Math. Phys. 61, 082105 (2020)

[31] G. Bastard, Wave mechanics applied to semiconductor heterostructures, Editions de Physique, Les Ulis, France, 1988)

[32] G. Barbagiovannia, D. Lockwoodb, R. N. Costa Filho, L. Goncharovad and P. Simpsond, Quantum confinement in Si and Ge nanostructures: Effect of crystallinity, Proc. SPIE 8915, Photonics North (2013), 891515 DOI: 10.1117/12.2036323

[33] E. Barbagiovanni, S. Cosentino, D. Lockwood, R. N. Costa Filho, A. Terrasi, and S. Mirabella, Influence of interface potential on the effective mass in Ge nanostructures, Journal of Applied Physics, 117, 154304 (2015)

[34] R. Szabo, Quantum field theory on noncommutative spaces, Phys.Rept. 378, 207 (2003)

[35] F. Delduc, Q. Duret, F. Gieres, M. Lefrancois, Magnetic fields in noncommutative quantum mechanics, J. Phys: Conf. Ser. 103, 012020 (2008)

[36] Richard J. Szabo, Quantum field theory on noncommutative spaces Physics Reports Volume 378, Issue 4, May 2003, Pages 207-299

[37] D. Bigatti and S. Susskind, Magnetic fields, branes and noncommutative geometry Phys. Rev. D 62, 066004 (2000)

[38] C. Bender,Making sense of non-Hermitian Hamiltonians, Rept. Prog. Phys. 70, 947-1018 (2007)

[39] A. Mostafazadeh, Pseudo-Hermiticity versus PT symmetry. The necessary condition for the reality of the spectrum, J. Math. Phys. 43, 205-214 (2002)

[40] F. Scholz, H. Geyer, and F. Hahne, Quasi-Hermitian operators in quantum mechanics and variational principle, Ann. Phys. 213, 74 (1992) 
[41] J. Dieudonné, Quasi-Hermitian operators, Proceedings of the International Symposium on Linear Spaces, Jerusalem 1960, Pergamon, Oxford, 115-122 (1961)

[42] M. Froissart, Covariant formalism of a field with indefinite metric, II Nuovo Cimento 14, 197-204 (1959)

[43] E. Sudarshan, Quantum Mechanical Systems with Indefinite Metric. I, Phys. Rev. 123, 2183-2193 (1961)

[44] A. Mostafazadeh, Pseudo-Hermiticity versus PT symmetry. The necessary condition for the reality of the spectrum, J. Math. Phys. 43, 205-214 (2002).

[45] D. Gao and M. Zhan, Constraining the generalized uncertainty principle with cold atoms, Phys. Rev. A 94, 013607 (2016).

[46] Jonas Schmöle, Mathias Dragosits, Hans Hepach and Markus Aspelmeyer, A micromechanical proof-of-principle experiment for measuring the gravitational force of milligram masses, Class. Quantum Grav. 33, 125031 (2016)

[47] K. Nozari and A. Etemadi, Minimal length, maximal momentum and Hilbert space representation of quantum mechanics, Phys. Rev. D 85, 104029 (2012)

[48] E. Borges, A possible deformed algebra and calculus inspired in nonextensive thermostatistics, Physica A 340, 95 (2004).

[49] C. Tsallis, Introduction to Nonextensive Statistical Mechanics: Approaching a Complex World, (Springer, New York, 2009)

[50] G. Kaniadakis and A. Scarfone, A new one-parameter deformation of the exponential function, Physica A 296, 405 (2001).

[51] G. Kaniadakis, Statistical mechanics in the context of special relativity, Phys. Rev. E 66, 056125 (2002).

[52] G. Kaniadakis, Statistical mechanics in the context of special relativity. II Phys. Rev. E 72, 036108 (2005) 